\title{
Descripción de posibles híbridos naturales entre Nothofagus pumilio y N. antarctica en Patagonia Sur (Argentina)
}

\author{
Description of possible natural hybrids between Nothofagus pumilio and N. antarctica at \\ South Patagonia (Argentina)
}

\section{Sarah Lilian Burns ${ }^{\mathrm{a} *}$, Juan Manuel Cellinia ${ }^{\mathrm{a}}$ María Vanessa Lencinas ${ }^{\mathrm{b}}$, Guillermo José Martínez Pastur ${ }^{\mathrm{b}}$, Stella Maris Rivera ${ }^{a}$}

*Autor de correspondencia: ${ }^{a}$ Universidad Nacional de La Plata, Facultad de Ciencias Agrarias y Forestales, CC 31 (1900)

La Plata, Buenos Aires, Argentina, tel.: +54 (221) 4236616, fax +54 (221) 425-2346, sarahlilburns@ gmail.com

bCADIC-CONICET, Ushuaia, Tierra del Fuego, Argentina.

\begin{abstract}
SUMMARY
The Nothofagus genus includes about 35 native tree species of the Southern hemisphere. In Patagonia various natural hybrids among different species of the genus were found. The aim of this work was to describe and determine the presence of putative natural hybrids between N. pumilio and N. antarctica at South Patagonia, through the study of morphological leaf characteristics. Twenty possible hybrids (individuals with both parental species characteristics), 10 pure individuals of each of the two parental species, were studied. Material was collected in transition areas between N. pumilio and N. antarctica in Tierra del Fuego forests. Twenty leaves of each individual were selected through random sampling. Length, width, distance from the largest width to the base, shape, base, apex and petiole length of each leaf were measured. The leaves of the supposed hybrid differ from those of $N$. pumilio in their size, distance of the largest width to the base and base. Nothofagus antarctica leaves are different from the supposed hybrid in their border, size and distance of the largest width to the base and base. These results prove that the studied species hybridize naturally in transition areas. This hybridization capacity can be used in genetic improvement programs for the development of individuals with the best characteristics of each parental species.
\end{abstract}

Key words: natural hybridization, Tierra del Fuego, leaf morphometry, genetic improvement.

\section{RESUMEN}

El género Nothofagus comprende alrededor de 35 especies nativas del Hemisferio Sur. En la Patagonia se han citado numerosos ejemplos de híbridos naturales entre varias especies de Nothofagus. El objetivo del presente trabajo fue analizar y describir la evidencia de la existencia de posibles híbridos naturales entre Nothofagus pumilio y N. antarctica en Patagonia Sur, mediante el estudio de caracteres morfológicos foliares. Se trabajó con 20 posibles híbridos recolectados en una zona de transición entre bosques de N. pumilio y N. antarctica de Tierra del Fuego, y con individuos de N. pumilio y N. antarctica obtenidos de poblaciones puras cercanas a las anteriores. Por medio de un muestreo aleatorio se seleccionaron 20 hojas de cada individuo a las que se les midió largo, ancho, distancia del mayor ancho a la base, forma, base, borde, ápice y largo del pecíolo. Las hojas de los posibles híbridos difirieron de $N$. pumilio en tamaño, distancia del ancho máximo a la base y frecuencia de asimetría en la base, y de $N$. antarctica en distancia del ancho máximo a la base, tamaño y frecuencias de asimetría en la base y de borde crenado. Los resultados obtenidos sugieren que $N$. pumilio y $N$. antarctica hibridizan naturalmente en zonas de condición ecotonal. Dicha capacidad de hibridación podría utilizarse en programas de mejoramiento genético para el desarrollo de individuos con las mejores características de cada especie parental.

Palabras clave: hibridación natural, Tierra del Fuego, morfometría foliar, mejoramiento genético.

\section{INTRODUCCIÓN}

El género Nothofagus comprende 35 especies arbóreas nativas del Hemisferio Sur (Vázquez y Rodríguez 1999). $\mathrm{Su}$ rango de distribución comprende el extremo sur de Sudamérica, Australia (incluida la isla de Tasmania), Nueva Zelandia, Nueva Caledonia, Nueva Guinea, y otras pequeñas islas oceánicas (Ramírez et al. 1997). En la República
Argentina este género está representado por seis especies: Nothofagus nervosa (Phil.) Dim. et Mil, N. obliqua (Mirb.) Blume., N. dombeyi (Mirb.) Oerst., N. betuloides (Mirb.) Blume., N. antarctica (Forst. f.) Oerst. y N. pumilio (Poepp. et Endl.) Krasser. La Isla Grande de Tierra del Fuego se encuentra dentro de la distribución natural de las tres últimas especies mencionadas. En Patagonia se han encontrado híbridos naturales entre $N$. obliqua y $N$. nervosa 
(Donoso et al. 1990, Gallo 1995, Grant y Clement 2004), N. obiqua y N. glauca (Donoso y Landrum 1979), así como en $N$. betuloides y $N$. nitida (Donoso y Atienza 1984). Asimismo Stecconi et al. (2004) describen la ocurrencia de híbridos naturales entre $N$. antarctica y $N$. dombeyi. $\mathrm{Si}$ bien se ha sugerido que $N$. antarctica y $N$. pumilio podrían hibridizar en condiciones naturales (van Steenis 1953, Donoso 1987), solamente han sido descritos híbridos de estas dos especies en el noroeste de la Patagonia en poblaciones de altura (Quiroga et al. 2005). El uso de las características morfológicas foliares como descriptores de hibridación ha sido ampliamente utilizado en este género (Stecconi et al. 2004, Quiroga et al. 2005).

Nothofagus antarctica es un árbol que puede llegar a los $15 \mathrm{~m}$ de altura, pero también puede presentarse en forma de arbusto o achaparrado en el límite altitudinal. Sus hojas son caducas, subcoriáceas, asimétricas, con predominio de forma aovada, de 2-3 cm de largo, redondas en el ápice, y desigualmente cuneadas o subtruncadas en la base, borde lobulado u ondeado con numerosos dientes irregulares en el espacio comprendido entre dos nervios; glabras a excepción de pecíolos y nervaduras (Dimitri y Milano 1950). Nothofagus pumilio es un árbol que puede llegar a los $30 \mathrm{~m}$ de altura. Sus hojas también son caducas pero coriáceas, elíptico-ovadas a ovadas, de $2-3 \mathrm{~cm}$ de largo, ápice obtuso, borde crenado, provistas de una escotadura internervial, base levemente asimétrica, anchamente cuneada o truncada, glabras o pubescentes sobre todo a lo largo de las nervaduras y bordes; pecíolos de hasta $0,5 \mathrm{~cm}$ de largo (Dimitri y Milano 1950). En Tierra del Fuego, los bosques de $N$. antarctica se encuentran mayormente en la parte central de la isla, limitando al norte con la estepa patagónica y al sur con los bosques de $N$. pumilio (Collado 1999). Los bosques de N. pumilio se extienden al sur hasta el Cabo de Hornos, trepando por los faldeos de las montañas hasta una altitud de 650 m s.n.m. (Roig 1998, Roig et al. 2002). En el ecotono entre estos bosques es frecuente encontrar árboles con características intermedias entre estas dos especies.

La hibridación entre dos especies suele estar facilitada por su cercanía filogenética (Hill y Jordan 1993, Martin y Dowd 1993), que en el caso de N. antarctica y $N$. pumilio ha sido probada en varios estudios (Manos 1997, Jordan y Hill 1999). Además, la hibridación en Nothofagus parece ocurrir sólo entre especies que comparten el mismo tipo de polen (Gallo 2002, Stecconi et al. 2004). Es de esperar que cuando se solapan los períodos de floración de estas dos especies puras y en ausencia de barreras postcigóticas, no existan restricciones para el flujo génico interespecífico (Quiroga et al. 2005). Esto es particularmente relevante en gradientes altitudinales, donde se ha demostrado que N. pumilio presenta marcadas restricciones al flujo génico intraespecífico (Premoli 2004). El aislamiento reproductivo entre poblaciones ubicadas a distinta altitud permitiría un mayor intercambio genético interespecífico con individuos simpátricos de N. antarctica (Gallo 2002).
La ocurrencia de hibridación entre N. pumilio y N. antarctica en zonas ecotonales de Patagonia Sur también podría estar asociada a la existencia de "hábitat híbridos", los que tal como postula Anderson (1948) son necesarios para que un híbrido pueda establecerse y sobrevivir. Bajo este punto de vista, la supervivencia de los híbridos durante la etapa de establecimiento podría aumentar debido a una reducción de la competencia con las especies puras en esos sitios. Por otra parte, la presencia de dos ensambles genéticos diferentes preadaptados a dichas condiciones podría aumentar sus capacidades de desarrollo y supervivencia en dicho entorno. Como sugieren Quiroga et al. (2005), los eventos de hibridación natural entre $N$. pumilio y $N$. antarctica podrían ser más frecuentes que lo considerado hasta el presente.

El objetivo de este trabajo es analizar la evidencia de la existencia de posibles híbridos naturales entre $N$. pumilio y $N$. antarctica en Patagonia Sur mediante el estudio de los caracteres morfológicos foliares y describir la morfometría foliar de los posibles híbridos.

\section{MÉTODOS}

Ubicación. Se trabajó en un área de 200 ha cubierta por bosques de Nothofagus spp. en la estancia San Justo en

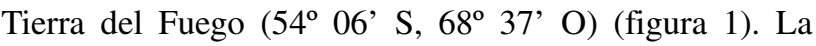
topografía del terreno se caracterizó por ser ondulada, con alternancia de vegas y cerros de hasta $500 \mathrm{~m}$ de altura, los cuales estuvieron mayormente cubiertos por bosques de $N$. pumilio y $N$. antarctica. Dentro del bosque se trabajó en la zona de contacto entre ambos tipos forestales de $N$. pumilio y $N$. antarctica, y en poblaciones puras de $N$. pumilio y $N$. antarctica alejadas $300 \mathrm{~m}$ de la zona de transición. El bosque muestreado de $N$. pumilio era un bosque primario en fase de envejecimiento, con alta cobertura de copas y estructura irregular. Por otro lado, el bosque de $N$. antarctica presentaba estructura irregular y con un cerramiento del dosel más abierto que el bosque de $N$. pumilio.

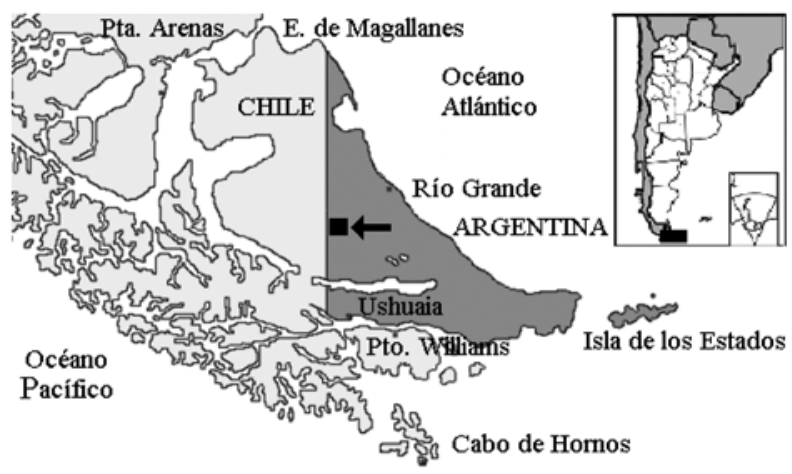

Figura 1. Ubicación de la zona bajo estudio en la provincia de Tierra del Fuego (Argentina).

Location of the studied zone in Tierra del Fuego province 
Selección de los árboles y procesamiento de muestras. Se seleccionaron 20 árboles de posibles híbridos en la zona de transición entre $N$. pumilio y $N$. antarctica, los que se eligieron por presentar características foliares intermedias entre ambas especies. Asimismo se seleccionaron 10 individuos de $N$. pumilio y 10 de $N$. antarctica creciendo en poblaciones puras. Todos los individuos seleccionados se encontraban en fase de envejecimiento (Schmidt y Urzúa 1982), correspondiendo en $N$. pumilio al rango de edades entre 120 y 250 años (Martínez Pastur et al. 2008), mientras que en $N$. antarctica el rango es de 111 a 220 años (Peri et al. 2006). El intervalo de edad de los posibles híbridos no fue determinado. A su vez eran dominantes en cuanto a su posición sociológica (posición relativa y tamaño de la copa de cada individuo en el estrato vertical). Tratando de captar la mayor variabilidad de forma y tamaño de hojas dentro de cada individuo, se recolectaron 10 hojas en la parte alta y 10 hojas en la parte inferior de la copa (Puntieri et al. 1999). Ya que existen diferencias morfológicas dadas por la ubicación dentro del brote (Puntieri et al. 2001), se seleccionaron las hojas a lo largo de todo el brote anual. La posición dentro del brote no fue considerada como variable. Una vez extraídas, las hojas fueron colocadas entre papeles para evitar la formación de pliegues, y se identificaron mediante rótulos acompañantes.

Variables analizadas. De cada individuo se midió el DAP (diámetro a 1,3 m de altura) mediante una cinta diamétrica. A todas las hojas se les midió el largo, el ancho, el largo del pecíolo y la distancia del ancho máximo a la base de la hoja, utilizando un calibre electrónico $( \pm 0,01 \mathrm{~mm})$. Utilizando la clasificación propuesta por Hickey (1974), se determinó la forma, la base, el borde y el ápice de la hoja. Además, se calcularon las siguientes relaciones: a) entre la distancia del ancho máximo a la base y el largo (RDL), y b) entre el largo y el ancho (RLA).

Análisis estadístico. Con los datos de DAP y morfología foliar se realizaron regresiones lineales simples con el fin de utilizar los residuos en un análisis de varianza. Se consideró como factor principal a las especies o al posible híbrido anidando las variables morfológicas foliares dentro de cada árbol. De esta manera el DAP se utilizó como covariable en el análisis de varianza, mediante la prueba de F, utilizando la prueba de Tukey para separar las medias. La probabilidad de rechazo fue $P<0,05$. Los datos se estandarizaron restándoles la media por especie y dividiéndolos por el desvío estándar. Con los datos estandarizados de las variables longitud del pecíolo, ancho, largo, distancia del ancho máximo a la base, base y borde de las hojas de $N$. pumilio, $N$. antarctica y el posible híbrido, se llevó a cabo un análisis de componentes principales (PCA). Luego, a partir de los valores para cada observación obtenidos en los tres primeros ejes del PCA (variables independientes), se realizó un análisis de discriminantes buscando obtener una función que separara eficientemente las especies puras de los posibles híbridos (variables de agrupamiento). El estadístico Wilks'Lambda se utilizó para evaluar el grado de separación entre los grupos.

\section{RESULTADOS}

La frecuencia de observación de los distintos rasgos de morfología foliar fueron diferentes en $N$. antarctica, $N$. pumilio y el posible híbrido. La forma del ápice, tanto en $N$. pumilio como $N$. antarctica fue siempre emarginada (cuadro 1), mientras que las hojas del posible híbrido presentaron un $9,4 \%$ de otras formas en el ápice (retusos, agudos y obtusos). En el análisis de la base foliar, el posible híbrido presentó una mayor frecuencia de asimetrías $(85,9 \%)$ que $N$. antarctica y $N$. pumilio, aunque en todos los casos las bases asimétricas fueron más comunes que las simétricas. En cuanto al borde, todas las hojas de $N$. pumilio y un $74,7 \%$ de las hojas del posible híbrido fueron crenadas, mientras que $N$. antarctica presentó sólo un 10,5\% de las hojas con borde crenado (cuadro 1 y figura 2).

En cuanto a la forma de las hojas se encontraron variaciones dentro de cada árbol para los tres grupos. La forma ovada fue la más frecuente, seguida por ovada angosta y ovada ancha (cuadro 2). El posible híbrido presentó un $5,3 \%$ de sus hojas lanceoladas y un $0,9 \%$ obovada ancha, característica que se presentó en una menor frecuencia en las otras especies.

Cuadro 1. Frecuencia de observación (\%) de distintas formas del ápice, bases foliares y bordes de las hojas de Nothofagus antarctica, $N$. pumilio y posibles híbridos. hybrid leaves.

Observation frequency (\%) for different shapes of apex, foliar base, and border in Nothofagus antarctica, N. pumilio and putative

\begin{tabular}{lcccccccr}
\hline \multirow{2}{*}{ Especie } & \multicolumn{3}{c}{ Forma del ápice } & \multicolumn{2}{c}{ Base foliar } & \multicolumn{2}{c}{ Borde de la hoja } \\
\cline { 2 - 9 } & Emarginado & Retuso & Agudo & Obtuso & Asimétrica & Simétrica & Crenado & Serrado \\
\hline N. antarctica & 100,0 & 0,0 & 0,0 & 0,0 & 65,5 & 34,5 & 10,5 & 89,5 \\
Híbrido & 90,6 & 6,0 & 2,8 & 0,6 & 85,9 & 14,1 & 74,7 & 25,3 \\
N. pumilio & 100,0 & 0,0 & 0,0 & 0,0 & 67,0 & 33,0 & 100,0 & 0,0 \\
\hline
\end{tabular}



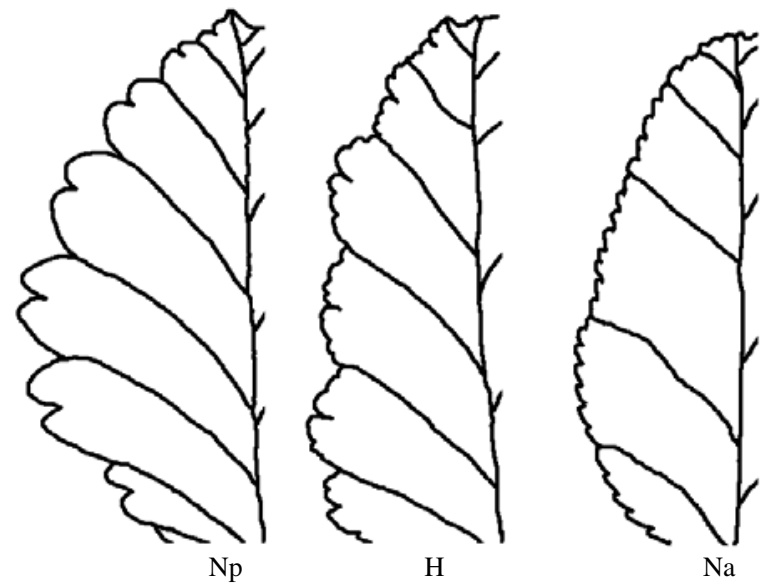

Figura 2. Diagrama comparativo de los diferentes bordes en las especies puras (Nothofagus antarctica y N. pumilio) y el posible híbrido. $\mathrm{H}=$ posible híbrido, $\mathrm{Np}=$ Nothofagus pumilio, $\mathrm{Na}=$ Nothofagus antarctica .

Comparative diagram of the different borders in the pure species (Nothofagus antarctica and N. pumilio) and the putative hybrid. $\mathrm{H}=$ putative hybrid, $\mathrm{Np}=$ Nothofagus pumilio, $\mathrm{Na}=$ Nothofagus antarctica.

Los modelos de regresión lineal simple entre el DAP y las variables de morfología foliar fueron significativos para todas las variables excepto RLA (cuadro 3).

Los resultados del análisis de varianza anidado con los residuos de las regresiones entre DAP y las distintas variables de morfometría foliar (cuadro 4) indicaron que la distancia del ancho máximo a la base, el largo, el ancho, la relación entre el largo y el ancho (RLA) y la relación entre la distancia del ancho máximo a la base y el largo (RDL) fueron significativamente diferentes entre las especies analizadas. El ancho, el largo y la distancia del ancho máximo a la base fueron significativamente diferentes entre las dos especies puras y el supuesto híbrido, siendo los valores de este último intermedios entre $N$. pumilio y $N$. antarctica. En la RDL $N$. antarctica se diferenció de $N$. pumilio y el posible híbrido, que fueron similares entre sí. Para la RLA sólo se observaron diferencias entre $N$. pumilio y el posible híbrido. Las diferencias estadísticas no fueron significativas para el largo del pecíolo.

En el análisis de componentes principales (figura 3) se observó una separación relativa entre $N$. pumilio y $N$. antarctica, ocupando el posible híbrido posiciones intermedias. El eje 1 fue determinado principalmente por el largo, el ancho y la distancia del máximo ancho a la base de las hojas (cuadro 5), mientras que en el eje 2 cobran más peso la forma del borde y la base de las hojas.

El análisis de discriminantes dio como resultado una función que explicó el $87 \%$ de la varianza total (Wilks' Lambda $=0,327$, aproximadamente $\mathrm{F}(6,123)=153,31$, $P<0,001)$. La clasificación general obtenida con el análisis de discriminantes indicó que 78\% de las muestras estuvieron clasificadas correctamente (cuadro 6). El porcentaje más bajo de clasificación se observó en el posible híbrido, lo que podría explicarse por la mayor semejanza del mismo con sus especies parentales, que originaría la mayor dispersión de los censos (figura 4). En el gráfico también se observó que algunos individuos de $N$. antarctica presentaron características similares a $N$. pumilio, principalmente debido a la presencia del borde crenado.

Cuadro 2. Frecuencia de observación (\%) de distintas formas de las hojas de Nothofagus antarctica, N. pumilio y posibles híbridos.

Observation frequency (\%) for different leaf shapes in Nothofagus antarctica, N. pumilio and putative hybrid leaves.

\begin{tabular}{lcccccccccc}
\hline Especie & E & EA & L & OV-AC & OV-AG & OV & OB-AC & OB-AG & OV-M-AC & SO \\
\hline N. antarctica & 0,5 & 1,0 & 2,0 & 15,5 & 30,0 & 47,0 & 0,0 & 0,0 & 4,0 & 0,0 \\
Híbrido & 0,9 & 1,6 & 5,3 & 18,4 & 28,1 & 39,7 & 0,9 & 1,6 & 3,1 & 0,3 \\
N. pumilio & 1,0 & 6,0 & 1,0 & 6,0 & 9,0 & 71,0 & 0,0 & 6,0 & 0,0 & 0,0 \\
\hline
\end{tabular}

$\mathrm{E}=$ elíptica; $\mathrm{EA}=$ elíptica ancha; $\mathrm{L}=$ lanceolada; OV-AC = ovada ancha; OV-AG = ovada angosta; OV = ovada; OB-AC = obovada ancha; $\mathrm{OB}-\mathrm{AG}=$ obovada angosta; OV-M-AC = ovada muy ancha; $\mathrm{SO}=$ suborbiculada.

Cuadro 3. Modelos de regresión lineal simple entre el DAP y las variables de morfología foliar. Simple linear regression models between DBH and leaf morphology variables.

\begin{tabular}{lcccccc}
\hline Parámetro & Pecíolo & D & Largo & Ancho & RDL & RLA \\
\hline Ordenada al origen & 3,2626 & 2,5773 & 11,5889 & 7,8545 & 0,293643 & 1,4717 \\
Pendiente & 0,0200 & 0,1000 & 0,1526 & 0,1170 & 0,001821 & $-0,0008$ \\
$\mathrm{R}^{2}$ Ajustado & 0,0698 & 0,3187 & 0,2009 & 0,2174 & 0,1406 & 0,0006 \\
\hline $\mathrm{F}$ & $47,471 * * *$ & $290,62 * * *$ & $156,59 * * *$ & $172,92 * * *$ & $102,29 * * *$ & $1,4025 \mathrm{~ns}$ \\
\hline
\end{tabular}

$\mathrm{D}$ = distancia del ancho máximo a la base; RDL = relación entre la distancia del ancho máximo a la base y el largo; RLA = relación entre el largo y el ancho. $\mathrm{F}=$ prueba de Fisher. El nivel de significancia de las pruebas estadísticas se indica como $* *=P<0,01 ; * * *=P<0,001 ; \mathrm{ns}=$ no significativo. 
Cuadro 4. Análisis de varianza para distintas medidas foliares de hojas de Nothofagus antarctica, N. pumilio y posibles híbridos utilizando el DAP como covariable.

ANOVA results for different leaf characteristics in Nothofagus antarctica, $N$. pumilio and putative hybrid leaves using DBH as a covariable.

\begin{tabular}{lcccccc}
\hline Especie & Pecíolo & $\mathrm{D}$ & Largo & Ancho & RDL & RLA \\
\hline N. antarctica & $3,8 \pm 0,30 \mathrm{a}$ & $4,9 \pm 0,10 \mathrm{a}$ & $15,2 \pm 1,20 \mathrm{a}$ & $10,7 \pm 0,90 \mathrm{a}$ & $0,3 \pm 0,01 \mathrm{~b}$ & $1,4 \pm 0,04 \mathrm{ab}$ \\
Híbrido & $4,0 \pm 0,20 \mathrm{a}$ & $6,3 \pm 0,10 \mathrm{~b}$ & $17,1 \pm 1,00 \mathrm{~b}$ & $12,0 \pm 0,70 \mathrm{~b}$ & $0,4 \pm 0,01 \mathrm{a}$ & $1,5 \pm 0,04 \mathrm{~b}$ \\
$N$. pumilio & $4,9 \pm 0,40 \mathrm{a}$ & $12,1 \pm 0,20 \mathrm{c}$ & $25,1 \pm 1,70 \mathrm{c}$ & $18,2 \pm 1,20 \mathrm{c}$ & $0,4 \pm 0,02 \mathrm{a}$ & $1,4 \pm 0,06 \mathrm{a}$ \\
\hline F & $2,74 \mathrm{~ns}$ & $305,08 * * *$ & $11,22 * * *$ & $12,73 * * *$ & $19,91 * * *$ & $0,48 \mathrm{~ns}$ \\
\hline
\end{tabular}

$\mathrm{D}=$ distancia del ancho máximo a la base $; \mathrm{RDL}=$ relación entre la distancia del ancho máximo a la base y el largo; RLA = relación entre el largo y el ancho. $\mathrm{F}=$ prueba de Fisher. El nivel de significancia de las pruebas estadísticas se indica como $* *=P<0,01 ; * * *=P<0,001$; ns $=$ no significativo. Las letras distintas en una misma columna indican diferencias significativas entre las medias por la prueba de Tukey al $5 \%$.

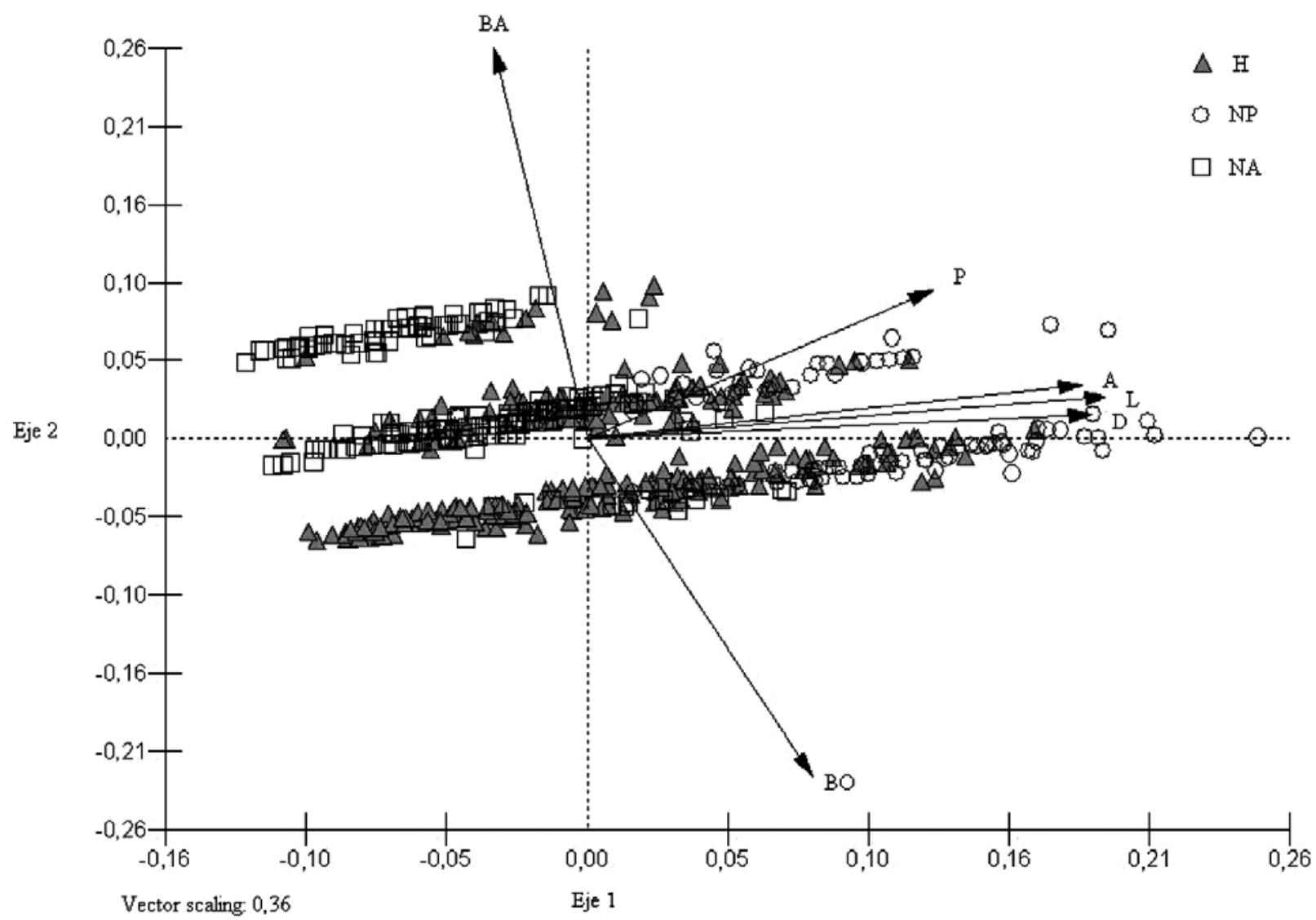

Figura 3. Gráfico de ordenamiento del análisis de componentes principales (ejes 1 y 2) con vectores que representan a las variables analizadas. $\mathrm{H}=$ posible híbrido, $\mathrm{NP}=N$. pumilio, $\mathrm{NA}=N$. antarctica . $\mathrm{BA}=$ base, $\mathrm{P}=$ largo del pecíolo, $\mathrm{A}=$ ancho, $\mathrm{L}=$ largo, $\mathrm{D}=$ distancia del ancho máximo a la base, $\mathrm{BO}=$ borde.

Principal Component Analysis ordination graphic (axes 1 and 2) with vectors that represent the analyzed variables. $\mathrm{H}=$ putative hybrid, $\mathrm{L}=$ Nothofagus pumilio, $\mathrm{N}=$ Nothofagus antarctica $. \mathrm{BA}=$ base, $\mathrm{P}=$ petiolar length, $\mathrm{A}=$ width, $\mathrm{L}=$ length, $\mathrm{D}=$ distance from the maximum width to the base, $\mathrm{BO}=$ edge width. 
Cuadro 5. Matriz de autovectores del análisis de componentes principales (PCA).

Eigenvalue matrix of the Principal Component Analysis (PCA).

\begin{tabular}{lrrr}
\hline Variables & Eje 1 & Eje 2 & Eje 3 \\
\hline Largo & 0,535 & 0,078 & $-0,008$ \\
Ancho & 0,508 & 0,099 & 0,150 \\
Distancia del ancho máximo a la base & 0,518 & 0,044 & 0,076 \\
Borde & 0,230 & $-0,622$ & 0,554 \\
Base & $-0,095$ & 0,721 & 0,645 \\
Largo del pecíolo & 0,354 & 0,275 & $-0,500$ \\
\hline
\end{tabular}

Cuadro 6. Clasificación obtenida por el análisis de discriminantes, basado en los valores de los ejes del PCA, utilizando a las especies puras (Nothofagus pumilio y N. antarctica) y al posible híbrido como grupos (porcentaje correcto entre paréntesis).

Classification obtained by discriminative analysis, based in PCA axis values, and using pure species (Nothofagus pumilio and $N$. antarctica) and the possible hybrid as groups (right percentage between parentheses).

\begin{tabular}{lccc}
\hline Grupo & N. antarctica & Híbrido & N. pumilio \\
\hline N. antarctica & $172(86 \%)$ & $26(13 \%)$ & $2(1 \%)$ \\
Híbrido & $65(20 \%)$ & $228(71 \%)$ & $27(9 \%)$ \\
N. pumilio & $0(0 \%)$ & $16(16 \%)$ & $84(84 \%)$ \\
\hline
\end{tabular}

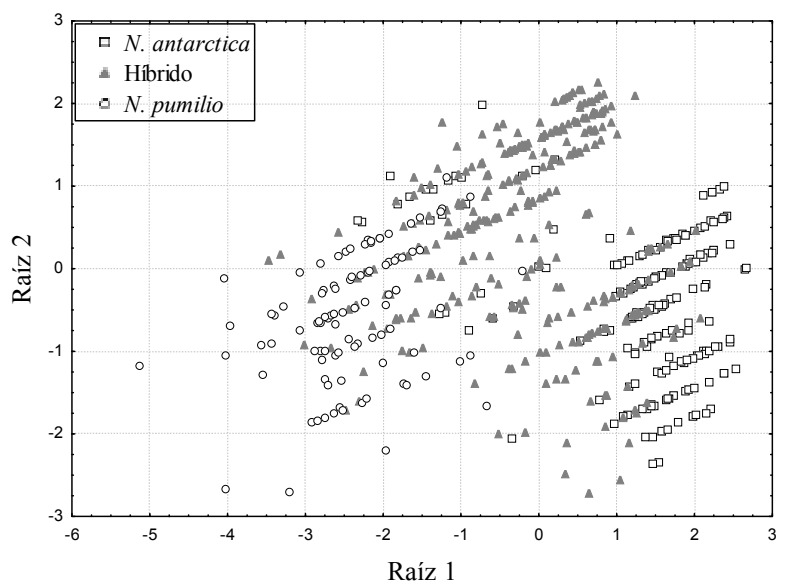

Figura 4. Gráfico de la capacidad discriminante de la clasificación previamente definida con los valores de los tres primeros ejes del PCA estandarizado para la separación de las especies puras (N. antarctica y N. pumilio) y el posible híbrido.

Discriminative analysis ordination graphic realized with three first axes of standardized PCA for separation of pure species (Nothofagus antarctica and $N$. pumilio) and the putative hybrid.

\section{DISCUSIÓN}

Los resultados de morfología foliar presentados en este trabajo muestran evidencias de la hibridación natural entre $N$. pumilio y $N$. antarctica en poblaciones naturales ecotonales de Patagonia Sur. La identificación de híbridos por medio de caracteres morfológicos se basa en el supuesto de que dichos individuos presentan fenotipos intermedios a los propios de las especies parentales, especialmente bajo regímenes de selección estabilizadora (Solbrig y Solbrig 1979). En este estudio, esta condición se observa respecto del borde de la hoja, que en el híbrido es intermedia entre el crenado de $N$. pumilio, y el serrado de $N$. antarctica (figura 2). Diferencias en el tamaño de las especies pueden influir en la morfología foliar. La utilización del DAP como covariable permitió identificar diferencias en los caracteres morfológicos debido a diferencias entre especies y no debidas al tamaño. La semejanza entre los individuos híbridos y una de sus especies parentales suele depender del grado de recombinación y de la intensidad de selección (Solbrig y Solbrig, 1979). La similitud entre el híbrido y $N$. pumilio estaría dada principalmente por la mayor frecuencia de hojas con borde crenado y la forma de las hojas así como en la RDL, mientras que difirió de esta especie parental en todas las otras variables estudiadas. Asimismo, se asemejó a $N$. antarctica en la RLA. Esto también fue observado por Quiroga et al. (2005) para otras variables de morfometría foliar.

El efecto de la dominancia (Rieseberg y Ellstrand 1993) provoca la manifestación de un mosaico de caracteres morfológicos parentales e intermedios en la primera generación híbrida. Esto también es sugerido por Allendorf et al. (2001), y se evidencia, en este trabajo, en la mayor diversidad de formas de ápice que presentan los posibles híbridos en relación a la de los ejemplares puros $-6 \%$, $2,8 \%$ y $0,6 \%$ de ápices retusos, agudos y obtusos, respectivamente- presentes en el híbrido, que no se presentaron en las especies parentales (cuadro 1). Es probable que las elevadas variaciones intraespecíficas de las especies parentales, que se reflejan en la amplia dispersión de puntos obtenida en el PCA y que también fueran observadas por Quiroga et al (2005), contribuyan en gran medida a la mayor variabilidad de los híbridos.

Las variaciones intraespecíficas de N. pumilio y N. antarctica están asociadas a la amplia distribución latitudinal y altitudinal de ambas especies, que se manifiesta en diferentes formas de vida (desde arbóreas y arbustivas a subarbustivas) en el caso de $N$. antarctica (Ramírez et al. 1985, Premoli 1991), y en diferentes caracteres morfológicos, ecofisiológicos e izoenzimáticos asociados a gradientes altitudinales en el caso de N. pumilio (Premoli 2003, 2004). Los caracteres de morfometría foliar observados en este trabajo para las especies puras fueron mayormente coincidentes con los observados por otros autores (Dimitri y Milano 1950, Premoli 1991). En el caso de N. antarctica se encontró cierta discrepancia con las observaciones de Moore (1983), ya que no se encontraron solamente hojas con borde lobulado y numerosos dientes irregulares en el espacio comprendido entre dos nervios, sino también hojas con borde crenado. Si bien en este trabajo se utilizaron los caracteres morfológicos para el reconocimiento de híbridos, es necesario contar con marcadores genéticos específicos 
de especie para establecer fehacientemente la identidad híbrida de un individuo (Gallo et al. 1997).

El número de individuos híbridos y el número de plantas puras analizadas en este trabajo es relativamente bajo. Por lo tanto, se requieren más estudios que analicen la ocurrencia de este fenómeno especialmente en otras áreas donde las dos especies coexisten en simpatría. Asimismo, el hecho de no haber considerado la ubicación de la hoja a lo largo del brote, es decir, sin haber estratificado el muestreo (proximal, media y distal), aumentaría la variabilidad observada dentro de las especies, dificultando la separación entre especies.

La existencia de híbridos naturales entre $N$. pumilio y $N$. antarctica es de gran importancia desde el punto de vista silvícola, ya que dicha capacidad de hibridación podría utilizarse en programas de mejoramiento genético para el desarrollo de individuos con las mejores características de cada especie parental, como la rectitud del fuste de $N$. pumilio, la dureza de la madera y la capacidad de desarrollo en un amplio rango de dispersión natural de $N$. antarctica. Además, ciertos parámetros del crecimiento pueden beneficiarse por la presencia de acervos genéticos diferentes, como lo demuestran estudios recientes sobre híbridos entre $N$. nervosa y N. obliqua, que han informado un mayor crecimiento en altura para éstos que para sus especies padres (Gallo et al. 1997), mejorando su potencial como productores de madera. Por otra parte, la hibridación natural de los Nothofagus spp. permitiría en cierta medida mantener la variación genética intragenérica, como ha sido recientemente discutido por Gallo (2002).

\section{CONCLUSIONES}

Los resultados obtenidos sugieren que $N$. pumilio y $N$. antarctica hibridizan naturalmente en Patagonia Sur en zonas de condición ecotonal, siendo de gran importancia y perspectivas silviculturales, ya que podría utilizarse la capacidad de hibridación en programas de mejoramiento genético para el desarrollo de individuos con las mejores características de cada especie parental, como la rectitud del fuste de $N$. pumilio, la dureza de la madera y la capacidad de desarrollo en su rango de dispersión natural de $N$. antarctica. Es importante tener en cuenta que la recolección de semillas de las especies puras debería realizarse evitando las zonas de borde, aspecto importante de considerar para los programas de propagación.

\section{AGRADECIMIENTOS}

Los autores desearían expresar su agradecimiento al Ing. Ignacio Mundo y al Dr. Marcelo Arturi por su colaboración y sus comentarios constructivos, y al Ing. Ricardo Vukasovic por su ayuda en la toma de las muestras.

\section{REFERENCIAS}

Allendorf FW, RF Leary, P Spruell, JK Wenburg. 2001. The problems with hybrids: setting conservation guidelines. Tree 16(11): 613-622.

Anderson E. 1948. Hybridization of the habitat. Evolution 2: $1-9$.

Collado L. 1999. Informe Técnico: Estratificación de los bosques fiscales de Tierra del Fuego mediante análisis de imágenes satelitales para el inventario forestal de la Provincia. Dirección de Bosques de Tierra del Fuego. Ushuaia, Argentina. 26 p. y 9 mapas.

Dimitri M, V Milano. 1950. Las plantas cultivadas en la República Argentina: Fagáceas. Min. Agric. Ganad. 8(156): 1-40.

Donoso C. 1987. Variación natural en especies de Nothofagus en Chile. Bosque 8(2): 85-97.

Donoso C, L Landrum. 1979. Nothofagus leoni Espinosa, a natural hybrid between $N$. obliqua (Mirb.) Oerst. and Nothofagus glauca (Phil.) Krasser. New Zealand Journal of Botany 17: 353-60.

Donoso C, JA Atienza. 1984. Hibridación natural entre Nothofagus betuloides (Mirb.) Oerst. y Nothofagus nitida (Phil.) Krasser. Medio Ambiente 7(1): 9-16.

Donoso C, J Morales, M Romero. 1990. Hibridación natural entre roble (Nothofagus alpina (Mirb.) Oerst.) y raulí (Nothofagus alpina (Poepp. et Endl.) Oerst.), en bosques del sur de Chile. Revista Chilena de Historia Natural 63: 49-60.

Gallo L. 1995. Posibles híbridos interespecíficos en progenies de raulí. In XXVI Congreso Argentino de Genética. San Carlos de Bariloche, 23-25 de octubre.

Gallo L, P Marchelli, A Breitembücher. 1997. Morphological and allozymic evidence of natural hybridization between two southern beeches (Nothofagus spp.) and its relation to heterozygosity and height growth. For. Genetics 4: 15-23.

Gallo L. 2002. Conceptual and experimental elements to model natural interspecific hybridization between two mountain southern beeches (Nothofagus spp.) In Degen B, MD Loveless, A Kremer eds. Modelling and experimental research on genetic processes in tropical and temperate forests. Kourou, French Guyane. Embrapa-Silvolab.

Grant ML, EJ Clement. 2004. Clarification of the name Nothofagus alpina and a new epithet for a Nothofagus hybrid. Bot. J. Linn. Soc. 146(4): 447-451.

Hickey LJ. 1974. Clasificación de la arquitectura de las hojas de Dicotiledóneas. Bol. Soc. Argent. Bot. 16(1-2): 1-26.

Hill RS, GJ Jordan. 1993. The evolutionary history of Nothofagus (Nothofagaceae). Aust. Syst. Bot. 6: 111-126.

Jordan G, RS Hill. 1999. The phylogenetic affinities of Nothofagus (Nothogaceae) leaf fossils based on combined molecular and mophological data. Int. J. Plant Sci. 160: 1177-1188.

Manos PS. 1997. Systematics of Nothofagus (Nothofagaceae) based on rDNA spacer sequences (ITS): Taxonomic congruence with morphology and plastid sequences. Am. J. Bot. 84(8): 1137-1155.

Martin PG, JM Dowd. 1993. Using sequences of rbcL to study phylogeny and biogeography of Nothofagus species. Aust. Syst. Bot. 6: 441-447.

Martínez Pastur GJ, JM Cellini, MV Lencinas, PL Peri. 2008. Stand growth model using volume increment/basal area ratios. Journal of Forest Science 54(3): 102-108. 
Moore D. 1983. Flora of Tierra del Fuego. Anthony Nelson Missouri Botanical Garden. 395 p.

Peri PL, V Gargaglione, GJ Martínez Pastur. 2006. Dynamics of above- and below-ground biomass and nutrient accumulation in an age sequence of Nothofagus antarctica forest of Southern Patagonia. Forest Ecology and Management 233: $85-99$

Premoli AC. 1991. Morfología y capacidad germinativa en poblaciones de Nothofagus antarctica (Forster) Oerst. del noroeste andino patagónico. Bosque 12(2): 53-59.

Premoli AC. 2003. Isozyme polymorphisms provide evidence of clinal variation with elevation in Nothofagus pumilio. $J$ Heredity 94(3): 218-226.

Premoli AC. 2004. Variación en Nothofagus pumilio (Poepp. et Endl.) Krasser. In Donoso C, AC Premoli, L Gallo, R Ipinza. Variación en las especies arbóreas de los bosques templados de Chile y Argentina. Santiago, Chile. Universitaria. p. 145-172.

Puntieri J, E Raffaele, P Martínez, D Barthélémy, C Brion. 1999. Morphological and architectural features of young Nothofagus pumilio (Poepp. et Endl.) Krasser (Fagaceae). Bot. J. Linn. Soc. 130: 395-410.

Puntieri J, M Damascos, MS Souza. 2001. Tendencias ontogenéticas en el tamaño y la forma de las hojas de Nothofagus pumilio (Poepp. et Endl.)Krasser (Fagaceae). Ecología Austral. 11: 105-114.

Quiroga MP, R Vidal Russell, AC Premoli. 2005. Evidencia morfológica e isoenzimática de hibridación natural entre Nothofagus antarctica y N. pumilio en el noroeste patagónico. Bosque 26(2): 25-32.

Ramírez C, M Correa, H Figueroa, J San Martín. 1985. Variación del hábito y hábitat de Nothofagus antarctica en el centro sur de Chile. Bosque 6(2): 55-73.
Ramírez C, C San Martín, A Oyarzún, H Figueroa. 1997. Morphoecological study on the South American Species of the genus Nothofagus. Plant Ecol. 130: 101-109.

Rieseberg LH, NC Ellstrand. 1993. What can mophological and molecular markers tell us about plant hybridization? Crit. Rev. Plant Sci. 12: 213-241.

Roig FA. 1998. La Vegetación de la Patagonia. In Correa MN ed. Flora Patagónica. INTA Colección Científica 8(1): 48-166.

Roig FA, G Martínez Pastur, V Moyano, L Pinedo, MV Lencinas. 2002. Cambial activity, phenology and climate in Nothofagus pumilio forests of Tierra del Fuego, Argentina. Actas del Sixth International Conference on Dendrochronology. Québec, Canadá. 281 p.

Schmidt H, A Urzúa. 1982. Transformación y manejo de los bosques de lenga en Magallanes. Ciencias Agrícolas 11. Santiago, Chile. Universitaria. 62 p.

Solbrig OT, DJ Solbrig. 1979. Introduction to population biology and evolution. Londres, UK. Addison - Wesley Pub. $468 \mathrm{p}$.

Stecconi M, P Marchelli, J Puntieri, P Picca, L Gallo. 2004. Natural hybridization between a deciduous (Nothofagus antarctica, Nothofagaceae) and an evergreen (N. dombeyi) forest tree species: evidence from morphological and isoenzymatic traits. Ann. Bot. (Lond.) 94: 775-786.

van Steenis DGGJ. 1953. Results of the Archbold expeditions Papuan Nothofagus. J Arnold Arbor. 34: 300-375.

Vázquez F, R Rodríguez. 1999. A new subspecies and two new combinations of Nothofagus Blume (Nothofagaceae). Chile. Bot. J. Linn. Soc. 129: 75-83.

Recibido: 25.05 .09

Aceptado: 13.10 .09 